\title{
Neutrino events at IceCube and the Fermi bubbles
}

\author{
Cecilia Lunardini, ${ }^{1, *}$ Soebur Razzaque, ${ }^{2, \dagger}$ Kristopher T. Theodoseau, ${ }^{1, \$}$ and Lili Yang ${ }^{1, \S}$ \\ ${ }^{1}$ Department of Physics, Arizona State University, Tempe, Arizona 85287-1504, USA \\ ${ }^{2}$ Department of Physics, University of Johannesburg, P.O. Box 524, Auckland Park 2006, South Africa
}

(Received 6 December 2013; published 21 July 2014)

\begin{abstract}
We discuss the possibility that the IceCube neutrino telescope might be observing the Fermi bubbles. If the bubbles discovered in gamma rays originate from accelerated protons, they should be strong emitters of high energy ( $\gtrsim \mathrm{GeV})$ neutrinos. These neutrinos are detectable as showerlike or tracklike events at a $\mathrm{Km}^{3}$ neutrino observatory. For a primary cosmic ray flux with spectrum $\propto E^{-2.1}$ and cutoff energy at or above $10 \mathrm{PeV}$, the Fermi bubble flux substantially exceeds the atmospheric background, and could account for up to $\sim 4-5$ of the 28 events detected above $\sim 30 \mathrm{TeV}$ at IceCube. Running the detector for $\sim 5-7$ more years should be sufficient to discover this flux at high significance. For a primary cosmic ray flux with steeper spectrum, and/or lower cutoff energy, longer running times will be required to overcome the background.
\end{abstract}

DOI: 10.1103/PhysRevD.90.023016

PACS numbers: 95.85.Ry, 98.70.Rz, 98.70.Sa

Very recently, the study of the sky at high energy has received a new impulse by the IceCube observation of an excess of neutrino flux, relative to the atmospheric neutrino background, above $\sim 30 \mathrm{TeV}[1,2]$. Of a total of 28 events, 21 are showers (or "cascades"), mostly caused by electron and tau neutrinos. For the remaining seven events a muon track has been identified, thus indicating a muon neutrino scattering. Two of the shower events exceed $1 \mathrm{PeV}$ of deposited energy [1], while the other 26 events are below $\sim 250 \mathrm{TeV}$. The 28 events observed at IceCube are a milestone in the field of neutrino astronomy, and have triggered a feverish activity to understand their meaning and their physics potential.

When comparing the data to theoretical models of high energy neutrino fluxes, it is natural to expect that multiple sources might contribute to the observed signal. Although prompt atmospheric neutrinos could fit some of the data [3], distant astrophysical sources are the most natural explanation. Cosmological emitters would likely produce a uniform, diffuse flux, and the spatial distribution of the events is compatible with this hypothesis. Recent literature discusses the cases of gamma ray bursts [4] and their lowerpowered counterparts [5,6], starburst galaxies [7-9], cores of active galactic nuclei $[10,11]$ and active galaxies [12], as well as intergalactic shocks [13].

In addition to a diffuse extragalactic component, Galactic sources would appear as anisotropies, spatially correlated with the Galactic disk and bulge. Recent analyses suggested spatial correlation of the IceCube data with unidentified TeV Galactic sources [14], with the Galactic center $[15]$ and the Fermi bubbles $[15,16]$. Origination from known Galactic TeV sources [17], and from the galactic

\footnotetext{
*Cecilia.Lunardini@asu.edu

†srazzaque@uj.ac.za

Kristopher.Theodoseau@asu.edu

lyang54@asu.edu
}

plane in general $[18,19]$ has also been studied. Beyond the standard model, ideas include the decay of heavy relics (Galactic and extragalactic) [20,21] and new physics contributions to the neutrino cross sections [22].

The focus of this paper is to explore the detectability of the Fermi bubbles (FB) at IceCube. Discovered in 2009 by Fermi-LAT [23], the bubbles are extended gamma-ray sources of globular shape, protruding symmetrically out of the Galactic center (GC) up to a distance of $\sim 9 \mathrm{kpc}$. Their origin, and the production mechanism of gamma rays, are yet unknown. Leaving aside possible new physics [24-28], concentrated high rate of supernova activity near the GC $[29,30]$ or accretion of gas by the GC black hole at a high rate in recent past [23] are the two main scenarios for bubble formation. The observed gamma rays are created either due to Compton scattering by highly relativistic electrons or due to decays of neutral pions created by interactions of energetic baryons. In the baryonic hypothesis, the gamma ray flux from the bubbles should have a neutrino counterpart of similar magnitude [29,31], which should be detectable in muon tracks at a $\mathrm{Km}^{3}$ detector in the northern hemisphere [31]. Dedicated experimental work on this is in progress [32,33], and an upper limit has been placed by the ANTARES collaboration (see Fig. 2) [32].

For a southern hemisphere detector like IceCube, instead, the main signature of the bubbles should be showers, thanks to the reduced background and increased shower effective area of the detector for down-going neutrinos compared to tracks $[1,2]$. Here we present the first quantitative study of the shower as well as down-going track events expected from the Fermi bubbles, both as a possible interpretation of some of the IceCube data, and as prediction for future searches with enhanced detector configuration and exposure.

Seen from Earth, the Fermi bubbles appear as extended sources in the southern sky (Fig. 1) subtending a total solid angle $\Omega_{\mathrm{FB}} \simeq 0.808 \mathrm{sr}$ [23]. Interestingly, their gamma ray 

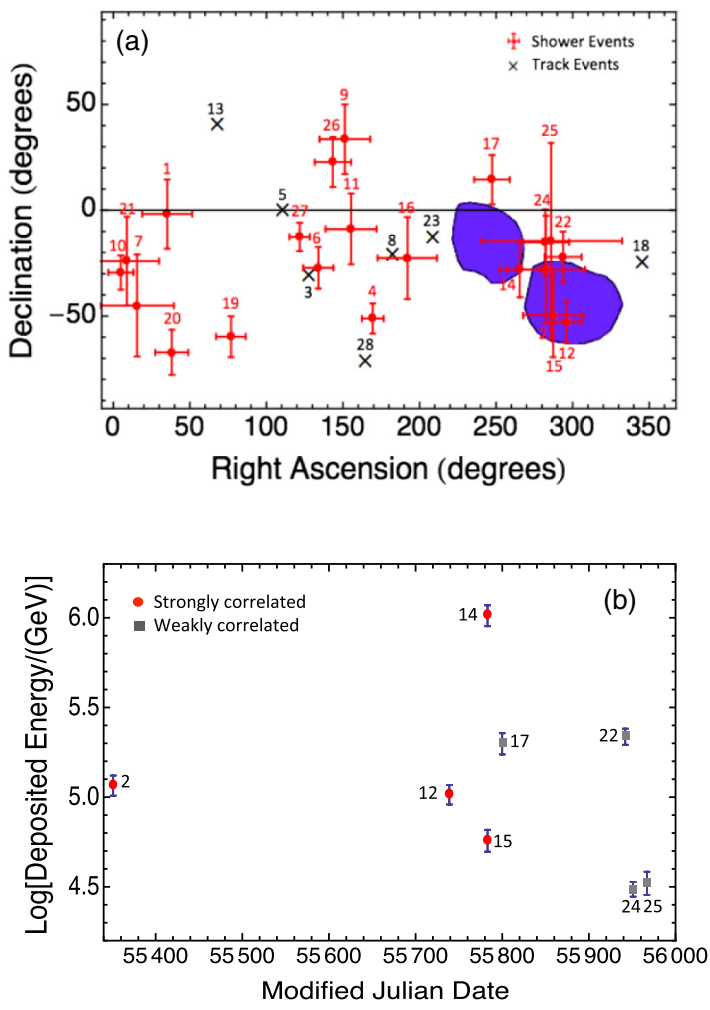

FIG. 1 (color online). (a) The IceCube events in equatorial coordinates, with their median angular errors, from [2]. The contours of the Fermi bubbles are shown as well. (b) The time and (deposited) energy distribution of the events that are spatially correlated with the bubbles.

emission per unit solid angle is roughly uniform over the extent of the bubbles [23], and the same feature is expected for the neutrino emission as well [31].

To estimate a possible correlation between the IceCube events and the FB, we compare the bubbles coordinates with the reconstructed coordinates of the IceCube events and their median angular errors [2], see Fig. 1. It appears that $N_{s}=4$ events (events number $2,12,14,15$ ) have their central position value inside the bubbles ("strongly correlated," meaning higher likelihood of originating from the FB), and $N_{w}=4$ (number 17, 22, 24, 25) are compatible with the bubbles within the error ("weakly correlated," or lower likelihood). Therefore $N=8$ is a conservative upper limit for the number of events from the FB, to be compared with theoretical predictions. Note that one of the strongly correlated events, event number 14 , has $\sim 1 \mathrm{PeV}$ of deposited energy [2].

To calculate the event rate in IceCube due to the FB, we use the neutrino fluxes from Ref. [31]. These fluxes are derived from fitting the gamma-ray data using $p p$ interactions of cosmic-ray protons in the bubbles with the ambient gas. A proton spectrum $d N / d E \propto E^{-k}$ was used, with a cutoff energy $E_{0}$, motivated by the maximum energy to which supernova remnants can accelerate cosmic ray protons. Theoretical estimates of $E_{0}$ vary from $1 \mathrm{PeV}$, at the
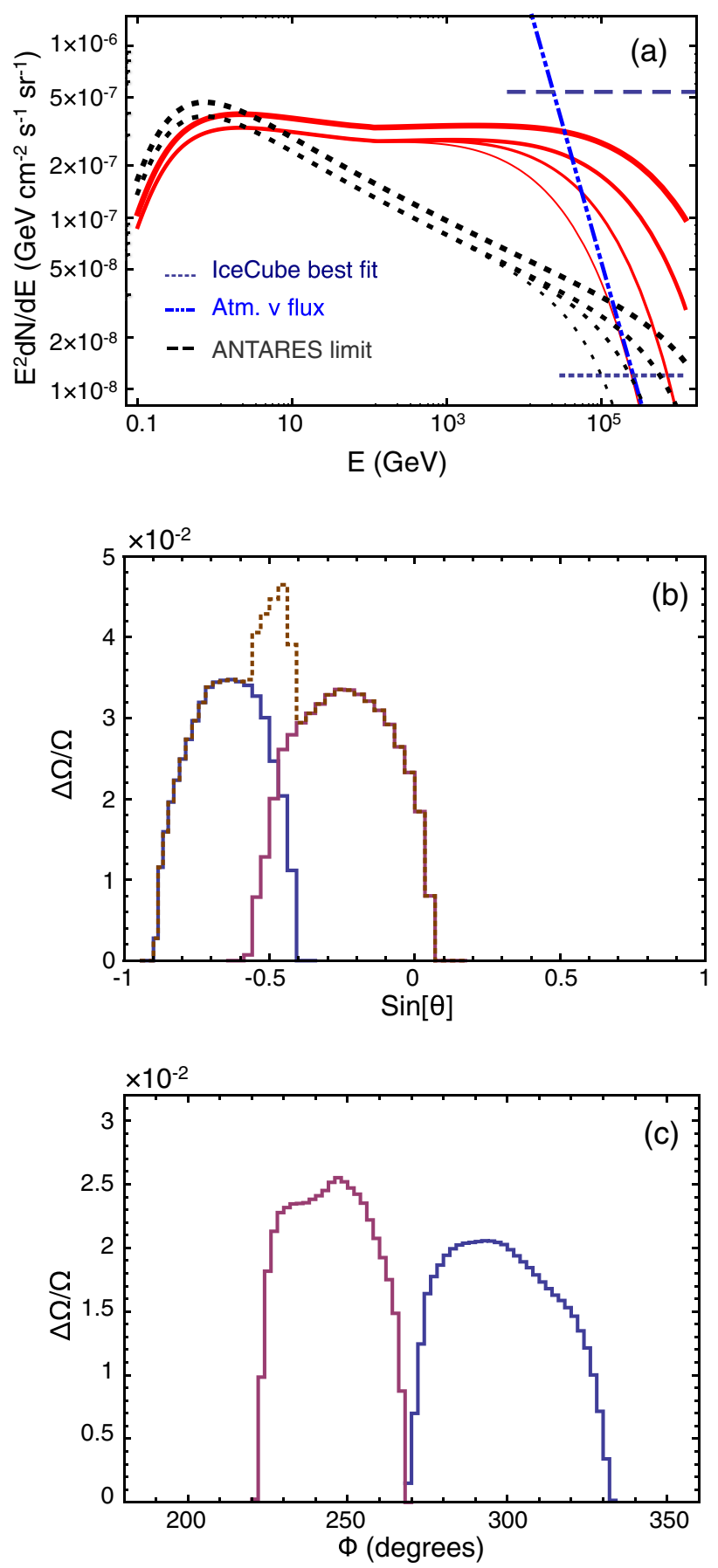

FIG. 2 (color online). (a) The expected $\nu_{\mu}+\bar{\nu}_{\mu}$ flux (solid lines) from the FB (before oscillations), normalized to the gamma-ray flux, as a function of the energy, for different proton spectral indices, $k$, and different cutoffs of the primary proton spectrum, $E_{0}$. Solid, red: $k=2.1$; dotted, black: $k=2.3$. For each we show, from thin to thick: $E_{0}=1,3,10,30 \mathrm{PeV}$. For comparison, we also show: (i) the atmospheric neutrino flux [34] averaged over $25^{\circ}-95^{\circ}$ zenith angle, (ii) the ANTARES upper limit [32] and (iii) the diffuse flux that best fits the IceCube data [2]. The other two panels show the distribution (normalized to 1 ) of the flux in $\sin \theta$ (with $\theta$ the declination angle) (b), and in the right ascension, $\phi$, (c), for each bubble (solid) and the total for both (dotted). 
"knee" of the cosmic-ray spectrum, to $100 \mathrm{PeV}$ [35]. The hard $\gamma$-ray spectrum of the FB is best represented with $k=2.1$, which is also favored by shock-acceleration theories. This is our default flux model unless otherwise specified. Given rather limited range of $\gamma$-ray data, a steeper $k=2.3$ proton spectrum is also compatible with observation. As shown in Fig. 2(a), the fluxes differ significantly above $\sim 200 \mathrm{GeV}$ (above the range of gamma-ray data) depending on $E_{0}$. Figure 2(a) also shows our most optimistic flux model (solid curve), obtained with $E_{0}=30 \mathrm{PeV}$, and a $\sim 20 \%$ increase of the normalization of the whole flux, which is allowed by the uncertainty in the gamma ray data. All results quoted for $E_{0}=30 \mathrm{PeV}$ will refer to this model. For comparison, in Fig. 2(a) we show the diffuse flux (at the detector after oscillation) that best fits the IceCube data [2]. Note that this flux refers to fitting the entire data sample in the assumption of a diffuse, uniform flux over the whole sky. It would be interesting to fit the data that are spatially correlated with the FB to find the level of flux required to reproduce them. At present, however, this cannot be done in the absence of more detailed information on the IceCube effective area and exposure.

The initial (preoscillation) flavor composition of the flux is $\nu_{e}: \nu_{\mu}: \nu_{\tau}=\epsilon: 1: 0$, with $\epsilon$ increasing from $\epsilon \simeq 0.57$ at $E=1 \mathrm{TeV}$ to $\epsilon \simeq 0.88$ at $E=1 \mathrm{PeV}$. This is explained by how energy is shared between the products of pion decay at different energies [36]. After oscillations (averaged vacuum oscillations, matter effects are negligible) the flavor ratios are close to $\nu_{e}: \nu_{\mu}: \nu_{\tau}=1: 1: 1$, with deviations up to $\sim 30 \%$ at $E \sim 1 \mathrm{PeV}$.

Because the emission is uniform over the FB surfaces, the fraction of flux in a solid angle bin, $\Delta F / F$ is given by the fraction of solid angle, $\Delta \Omega / \Omega_{\mathrm{FB}}$. This is shown in Figs. 2(b) and 2(c).

To establish the significance of the FB signal, one should consider the main backgrounds, i.e., atmospheric muons and atmospheric neutrinos. For the former, the background level depends on the detector veto, and could change with future technological advances. We refer to [2] for this. Here we model the atmospheric neutrino background using the neutrino flux prediction by Honda et al. [34] (which is a good fit of IceCube's atmospheric data [37]), extrapolated at high energy, and a $\nu_{\mu} / \nu_{e}$ ratio of about 14 [38]. We also consider the flux to be symmetric in $\cos \theta_{z}$ [39]. Oscillations are negligible at the energies and zenith angles of interest [40], therefore the $\nu_{\tau}$ atmospheric flux is neglected altogether. To account for the error on the direction of arrival of the neutrinos, we calculate the rate of atmospheric shower events over a solid angle larger than $\Omega_{\mathrm{FB}}$, obtained by encasing each bubble in a rectangle in the $\theta$ and $\phi$ coordinates, and then enlarge such rectangle by $\omega=15^{\circ}$ (motivated by the detector's angular resolution [2]) on each side. The total solid angle obtained in this way is $\Omega_{b c k g} \simeq 2.75 \mathrm{sr}$. For track events, where the angular resolution is less than a degree [2], the angle $\Omega_{\mathrm{FB}}$ is used. We find that showerlike and tracklike events contribute comparably to the total background rate, because the predominance of the $\nu_{\mu}$ species in the atmospheric flux compensates for the smaller effective area for tracks.

Figure 3(a) shows the expected number of signal and background events for $k=2.1$ and 10 years running time, above an energy threshold $E_{\mathrm{th}}$, as a function of $E_{\mathrm{th}}$. We observe that, for $E_{0} \gtrsim 10 \mathrm{PeV}$, the signal rises above the background, by up to $\sim 2$ orders of magnitude for the most optimistic flux model. Specifically, for $E_{0}=30 \mathrm{PeV}$ and $E_{\text {th }}=10^{4.6} \mathrm{GeV}$, we find 23 signal and two background events, amounting to a $\sim 4.4 \sigma$ excess due to the FB. For the same parameters, a significance of $3 \sigma$ would be obtained with about seven years of running time. The time needed for discovery might be shorter with the use of detailed statistical analyses of the spatial correlation with the bubbles, and/or if a compatible excess is observed in track events at a detector in the Northern hemisphere [31,33]. For the most conservative spectrum, $E_{0}=1 \mathrm{PeV}$, the background is
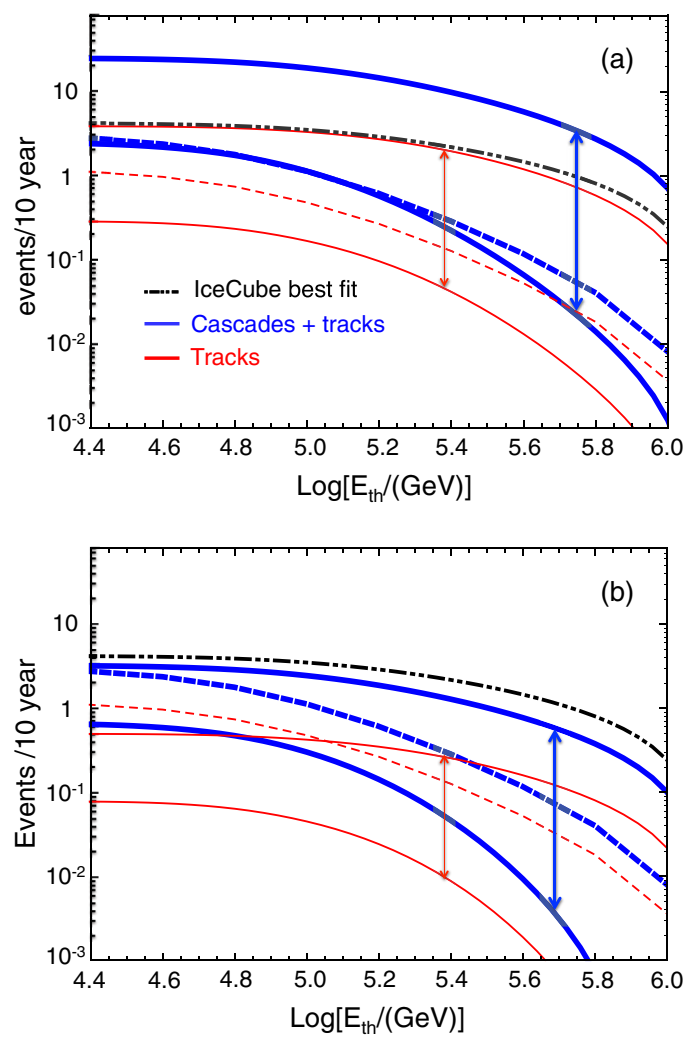

FIG. 3 (color online). Events expected at IceCube per decade, as a function of the neutrino energy threshold $E_{\mathrm{th}}$, for the primary proton spectrum index $k=2.1$ (upper panel) and $k=2.3$ (lower panel). Solid: FB signal, for the total of showerlike and tracklike events (thick) and for tracklike events only (thin). The arrows indicate the effect of varying the primary spectrum cutoff in the interval $E_{0}=1-30 \mathrm{PeV}$. Dashed: the same but from atmospheric fluxes. Dot-dot-dashed: showerlike and tracklike events from the IceCube best-fit flux in Fig. 2(a). 
comparable to the signal for all thresholds, therefore, detection prospects are poor. For the steeper spectrum, $k=$ 2.3 [Fig. 3(b)], conclusions are similar, overall. However, even for the most optimistic spectrum, the signal/background ratio is modest, and becomes significant only above $\sim 10^{5} \mathrm{GeV}$, where the event rate is small.

Figure 4 gives the distribution of signal and background events per decade in bins of neutrino energy [41]. The width of the bins is chosen such that in each bin the highest energy is 4 times the lowest energy, which is roughly the maximum uncertainty in reconstructing the neutrino energy from the deposited energy in case of neutral current interactions [42]. Overall, Fig. 4 confirms the results of Fig. 3(a); it also shows that most number of events are expected in the bin $\log (E / \mathrm{GeV})=5-5.6$, due to a sharp rise of the effective area below $\sim 1 \mathrm{PeV}$ [2] and an $E_{0^{-}}$ dependent exponential drop of the flux at high energies.

Let us now apply our results to the IceCube data, from the recent 662 days search [2]. Table I and Fig. 5 show the expected number of events for signal and background. For the total showerlike and tracklike events, less than one atmospheric background event is expected. The FB signal rises above one event for $E_{0}>3 \mathrm{PeV}$, and for $E_{0} \geq 10 \mathrm{PeV}$, it starts to be close to the measured rate. In particular, for $E_{0}=30 \mathrm{PeV}$, we expect $N \sim 3$ and $N \sim 1$
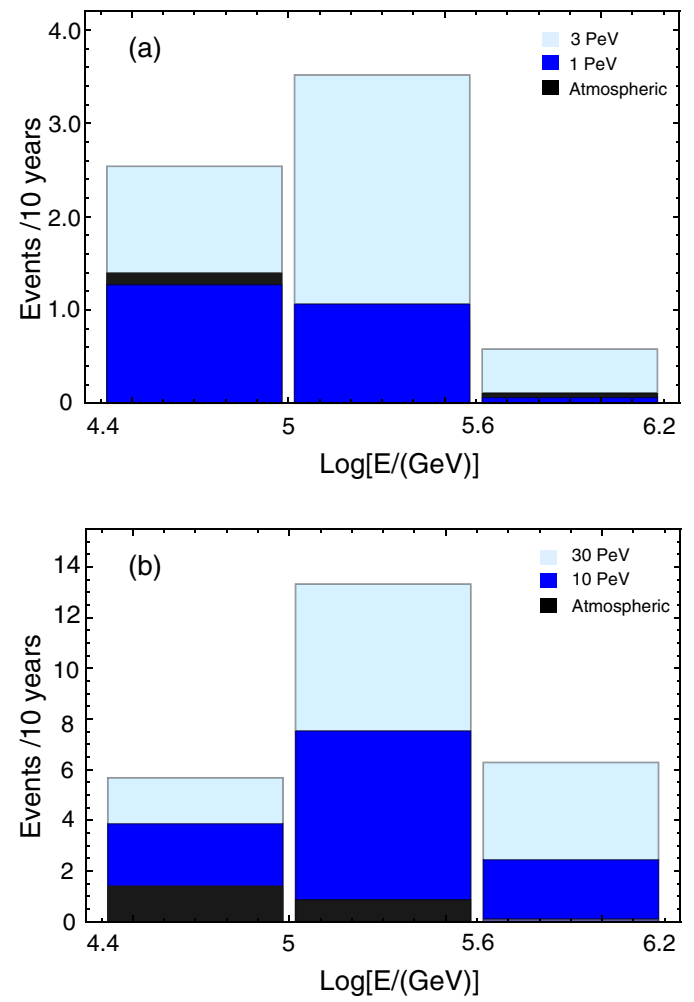

FIG. 4 (color online). Expected neutrino energy distribution of the total showerlike and tracklike events per decade, for signal (with $k=2.1$ ) and for atmospheric background. (a) $E_{0}=1,3 \mathrm{PeV}$; (b) $E_{0}=10,30 \mathrm{PeV}$. For $E_{0}=1 \mathrm{PeV}$, signal and background are very close in all bins.
TABLE I. Showers + tracklike events expected in three bins of neutrino energy, from the atmospheric background and from the FB (for different primary spectrum cutoff, $E_{0}$ ) for the 662 days IceCube search. The numbers in brackets refer to tracklike events only.

\begin{tabular}{lcccc}
\hline \hline & \multicolumn{5}{c}{$\log (E / \mathrm{GeV})$} & \\
& $4.4-5$ & $5-5.6$ & $5.6-6.2$ & Total \\
\hline$E_{0} / \mathrm{PeV}=1$ & 0.23 & 0.19 & 0.01 & 0.43 \\
& {$[0.02]$} & {$[0.03]$} & {$[0]$} & {$[0.05]$} \\
$E_{0} / \mathrm{PeV}=3$ & 0.46 & 0.64 & 0.11 & 1.2 \\
& {$[0.04]$} & {$[0.1]$} & {$[0.02]$} & {$[0.16]$} \\
$E_{0} / \mathrm{PeV}=10$ & 0.7 & 1.37 & 0.44 & 2.51 \\
& {$[0.07]$} & {$[0.21]$} & {$[0.09]$} & {$[0.37]$} \\
$E_{0} / \mathrm{PeV}=30$ & 1.03 & 2.42 & 1.14 & 4.59 \\
& {$[0.1]$} & {$[0.38]$} & {$[0.24]$} & {$[0.72]$} \\
Background & 0.25 & 0.16 & 0.02 & 0.43 \\
& {$[0.07]$} & {$[0.06]$} & {$[0.01]$} & {$[0.14]$} \\
\hline \hline
\end{tabular}

events below and above $E=10^{5.6} \mathrm{GeV} \simeq 400 \mathrm{TeV}$ of neutrino energy respectively. This is intriguingly close, in number and energy distribution, to the observation of the $N_{s}=4$ events strongly correlated with the FB (Fig. 5).

In synthesis, has IceCube already detected the FB? The answer might be yes, if the neutrino spectrum is relatively hard, coming from a primary proton flux that falls like $E^{-2.1}$ and has a cutoff above $10 \mathrm{PeV}$ or so. $E_{0} \simeq 10-30 \mathrm{PeV}$ seems to best fit the data, especially the events strongly correlated with the FB. Observation of a neutrino flux from the FB may provide clues to the maximum limit of particle acceleration in supernova remnants, which are thought to be the origin of energetic protons in the FB and which are not widely discussed as sources of cosmic rays above $1 \mathrm{PeV}$. Note, however, that protons (and in effect heavy

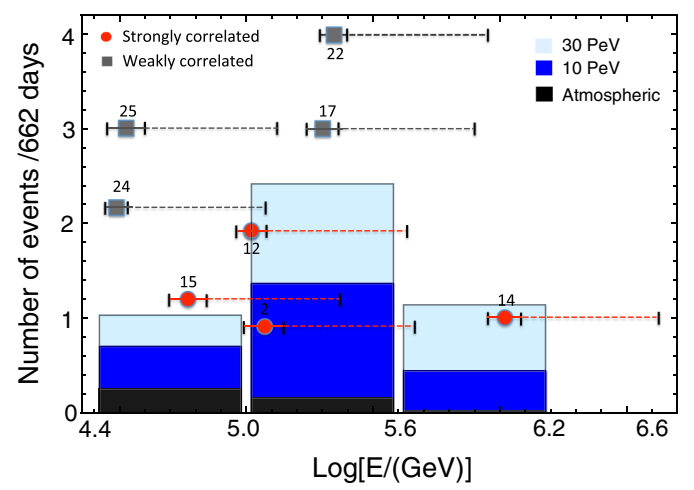

FIG. 5 (color online). The same as Fig. 4(b) for the IceCube running time of 662 days. The IceCube events that correlate with the FB (from Fig. 1) are shown for comparison. Their coordinates on the vertical axis give a visual representation of the number of events for which the central value of the observed energy falls in a given bin. The solid (dashed) error bars represent, respectively, the error on the observed energy and the factor of 3-4 difference between neutrino energy and observed energy for neutral current events. 
nuclei, if present) in the FB are thought to lose all their energy by $p p$ interactions over the lifetime of the bubbles (several billion years) in the hadronic model [29]. Thus, FB are not expected to contribute significantly to the observed cosmic-ray spectrum, which is dominated by heavy nuclei above the knee at $\sim 1 \mathrm{PeV}$.

Our model predicts that up to $\sim 5$ of the observed IceCube events might be due to the FB. Like other models with a strong Galactic contribution, this implies that the extragalactic, diffuse, flux required to explain the rest of the data should be lower compared to the case with no galactic flux. Considering that about $\sim 10$ events in IceCube are likely to be background [2], the diffuse flux normalization would have to be smaller by $\sim 4 /(28-10)=22 \%$. This figure is insignificant with the current statistics, but might be nevertheless important to consider when looking in perspective for the future.

With higher statistics, the FB should clearly manifest themselves with an excess of events correlated with their position and extent in the sky (Fig. 1). No other phenomenon would have such a signature. The statistics needed to have a significant detection of the FB depends on the level of the diffuse neutrino flux from other sources (other than atmospheric background), however, at least for the most optimistic scenario $\left(E_{0}=30 \mathrm{PeV}\right) 7-10$ years time should be sufficient, see Fig. 3.
The FB signal will be strongly substantiated by a northern hemisphere detector like the future $\mathrm{Km} 3 \mathrm{Net}$ [33], which will be at a nearly optimal location to look for tracklike events from the bubbles [31]. More than 300 events per decade are expected for $E_{0}=10 \mathrm{PeV}$. The complementarity of IceCube and $\mathrm{Km} 3 \mathrm{Net}$ will help to resolve a number of uncertainties and degeneracies (for example, the track events at $\mathrm{Km} 3 \mathrm{Net}$ will have a better angular resolution, thus helping to separate the FB from other possible Galactic sources).

If the FB are confirmed to be strong neutrino emitters, the implications on the physics of our galaxy would be profound. In particular, this would support the idea of a long time scale of the activity of the Galactic center, $\sim 10^{9}$ years. This is the time required to form the $\mathrm{FB}$ in the hadronic model [29], as opposed to the much shorter time (millions of years) required in a leptonic model of the bubbles.

We thank Albrecth Karle, Mariola Lesiak-Bzdak, Jakob van Santen and Nathan Whitehorn. C. L. and L. Y. acknowledge the National Science Foundation Grant No. PHY-1205745. K. T. acknowledges the ASU/NASA Space Grant 2013 for partial support. S. R. acknowledges support from the National Research Foundation (South Africa) Grant CPRR 2014 No. 87823.
[1] M. Aartsen et al., Phys. Rev. Lett. 111, 021103 (2013).

[2] M. Aartsen et al., Science 342, 1242856 (2013).

[3] P. Lipari, arXiv:1308.2086.

[4] S. Razzaque, Phys. Rev. D 88, 103003 (2013).

[5] S. Razzaque, P. Meszaros, and E. Waxman, Phys. Rev. D 68, 083001 (2003).

[6] K. Murase and K. Ioka, Phys. Rev. Lett. 111, 121102 (2013).

[7] A. Loeb and E. Waxman, J. Cosmol. Astropart. Phys. 05 (2006) 003.

[8] F. W. Stecker, Astropart. Phys. 26, 398 (2007).

[9] R.-Y. Liu, X.-Y. Wang, S. Inoue, R. Crocker, and F. Aharonian, Phys. Rev. D 89, 083004 (2014).

[10] F. W. Stecker, Phys. Rev. D 88, 047301 (2013).

[11] W. Winter, Phys. Rev. D 88, 083007 (2013).

[12] O. E. Kalashev, A. Kusenko, and W. Essey, Phys. Rev. Lett. 111, 041103 (2013).

[13] K. Murase, M. Ahlers, and B. C. Lacki, Phys. Rev. D 88, 121301 (2013).

[14] D. Fox, K. Kashiyama, and P. Meszaros, Astrophys. J. 774, 74 (2013).

[15] S. Razzaque, Phys. Rev. D 88, 081302 (2013).

[16] M. Ahlers and K. Murase, arXiv:1309.4077.

[17] M. Gonzalez-Garcia, F. Halzen, and V. Niro, Astropart. Phys. 57-58, 39 (2014).
[18] L. A. Anchordoqui, H. Goldberg, M. H. Lynch, A. V. Olinto, T. C. Paul, and T. J. Weiler, Phys. Rev. D 89, 083003 (2014).

[19] J. C. Joshi, W. Winter, and N. Gupta, Mon. Not. R. Astron. Soc. 439, 3414 (2014).

[20] B. Feldstein et al., Phys. Rev. D 88, 015004 (2013).

[21] A. Esmaili and P. D. Serpico, J. Cosmol. Astropart. Phys. 11 (2013) 054.

[22] V. Barger and W.-Y. Keung, Phys. Lett. B 727, 190 (2013).

[23] M. Su, T. R. Slatyer, and D. P. Finkbeiner, Astrophys. J. 724, 1044 (2010).

[24] I. Cholis, Phys. Rev. D 88, 063524 (2013).

[25] N. Okada and O. Seto, Phys. Rev. D 89, 043525 (2014).

[26] D. Hooper and T. R. Slatyer, Phys. Dark Univ. 2, 118 (2013).

[27] W.-C. Huang, A. Urbano, and W. Xue, arXiv:1307.6862.

[28] W.-C. Huang, A. Urbano, and W. Xue, J. Cosmol. Astropart. Phys. 04 (2014) 020.

[29] R. M. Crocker and F. Aharonian, Phys. Rev. Lett. 106, 101102 (2011).

[30] Y. Fujita, Y. Ohira, and R. Yamazaki, Astrophys. J. 775, L20 (2013).

[31] C. Lunardini and S. Razzaque, Phys. Rev. Lett. 108, 221102 (2012).

[32] S. Adrián-Martínez et al., Eur. Phys. J. C 74, 2701 (2014). 
[33] S. Adrian-Martinez et al., Astropart. Phys. 42, 7 (2013).

[34] M. Honda, T. Kajita, K. Kasahara, S. Midorikawa, and T. Sanuki, Phys. Rev. D 75, 043006 (2007).

[35] V. Ptuskin, V. Zirakashvili, and E. Seo, Astrophys. J. 718, 31 (2010).

[36] P. Lipari, Astropart. Phys. 1, 195 (1993).

[37] R. Abbasi et al., Phys. Rev. D 83, 012001 (2011).

[38] T. Sinegovskaya, E. Ogorodnikova, and S. Sinegovsky, arXiv:1306.5907.
[39] M. Sajjad Athar, M. Honda, T. Kajita, K. Kasahara, and S. Midorikawa, Phys. Lett. B 718, 1375 (2013).

[40] T. Gaisser and T. Stanev, Phys. Rev. D 57, 1977 (1998).

[41] The observed energy could be lower by a factor of 3-4 than the neutrino energy for neutral current events [42]. Therefore, our Fig. 4 is not representative of the actual observed data spectrum. However, it should be a reasonable approximation, considering our choice of very wide energy bins.

[42] M. G. Aartsen et al., JINST 9, P03009 (2014). 